\title{
A New Approach to Quantising Space-Time: III. State Vectors as Functions on Arrows
}

\author{
C.J. Isham \\ The Blackett Laboratory \\ Imperial College of Science, Technology \& Medicine \\ South Kensington \\ London SW7 2BZ \\ c.isham@imperial.ac.uk
}

\begin{abstract}
In [1], a new approach was suggested for quantising space-time, or space. This involved developing a procedure for quantising a system whose configuration space - or history-theory analogue - is the set of objects, $\mathbf{O b}(\mathcal{Q})$, in a (small) category $\mathcal{Q}$. The quantum states in this approach are cross-sections of a bundle $\boldsymbol{A} \rightsquigarrow \mathcal{K}[\boldsymbol{A}]$ of Hilbert spaces over $\mathrm{Ob}(\mathcal{Q})$. The Hilbert spaces $\mathcal{K}[\boldsymbol{A}], \boldsymbol{A} \in \mathrm{Ob}(\mathcal{Q})$, depend strongly on the object $\boldsymbol{A}$, and have to be chosen so as to get an irreducible, faithful, representation of the basic 'category quantisation monoid'. In the present paper, we develop a different approach in which the state vectors are complex-valued functions on the set of arrows in $\mathcal{Q}$. This throws a new light on the Hilbert bundle scheme: in particular, we recover the results of that approach in the, physically important, example when $\mathcal{Q}$ is a small category of finite sets.
\end{abstract}

\section{Introduction}

In [1] (hereafter referred to as I.) a new approach was developed for quantising space-time or space. This was based on the observation that such entities can be regarded as the objects in a category whose arrows are

e-print archive:

http://lanl.arXiv.org/abs/gr-qc/0306064 
structure-preserving maps. This lead to considering the general problem of finding the quantum theory of a system whose configuration space is the set of objects, $\mathrm{Ob}(\mathcal{Q})$, in a small ${ }^{1}$ category $\mathcal{Q}$. A key idea in $\mathbf{I}$. is to use the monoid $\operatorname{AF}(\mathcal{Q})$ of arrow fields on $\mathcal{Q}$ as an analogue of the collection of momentum variables in a normal quantum theory. Here, an 'arrow field' $X$ is defined to be an association of an arrow $X(A)$ to each object $A \in \mathrm{Ob}(\mathcal{Q})$ with the property that the domain of $X(A)$ is $A$.

The simplest scheme of this type is where the state vectors are complexvalued functions $A \mapsto \psi(A)$ on $\mathrm{Ob}(\mathcal{Q})$. The key operators are then defined by

$$
\begin{aligned}
(\hat{a}(X) \psi)(A) & :=\psi(\operatorname{Ran} X(A)) \\
(\hat{\beta} \psi)(A) & :=\beta(A) \psi(A)
\end{aligned}
$$

for all $A \in \mathrm{Ob}(\mathcal{Q})$. Here, $X$ is any arrow field, and $\beta$ is any element of the set $F(\mathrm{Ob}(\mathcal{Q}), \mathbb{R})$ of all real-valued functions on $\mathrm{Ob}(\mathcal{Q})$. We also define the exponentiated operator $\hat{V}(\beta):=\exp -i \hat{\beta}$ :

$$
(\hat{V}(\beta) \psi)(A):=e^{-i \beta(A)} \psi(A) .
$$

The operators $\hat{a}(X), X \in \operatorname{AF}(\mathcal{Q})$, and $\hat{V}(\beta), \beta \in F(\mathrm{Ob}(\mathcal{Q}), \mathbb{R})$, satisfy the equations

$$
\begin{aligned}
\hat{a}\left(X_{2}\right) \hat{a}\left(X_{1}\right) & =\hat{a}\left(X_{1} \& X_{2}\right) \\
\hat{V}\left(\beta_{1}\right) \hat{V}\left(\beta_{2}\right) & =\hat{V}\left(\beta_{1}+\beta_{2}\right) \\
\hat{a}(X) \hat{V}(\beta) & =\hat{V}\left(\beta \circ \ell_{X}\right) \hat{a}(X)
\end{aligned}
$$

where ' $\&$ ' denotes the multiplication operation (see Eq. (2.1)) in the monoid $\operatorname{AF}(\mathcal{Q})$, and $\ell_{X}$ is the action of the arrow field $X$ on the set of objects, defined by $\ell_{X} A:=\operatorname{Ran} X(A)$ for all $A \in \operatorname{Ob}(\mathcal{Q})$.

Equations (1.4-1.6) correspond to a representation of the 'category quantisation monoid' of $\mathcal{Q}$, defined to be the semi-direct product $\operatorname{AF}(\mathcal{Q}) \times_{\ell}$ $F(\mathrm{Ob}(\mathcal{Q}), \mathbb{R})$. In general, the possible quantisations of the system are deemed to be given by the irreducible, faithful representations of this monoid.

However, the simple representation given by Eqs. (1.1-1.2) is not faithful: in particular, it fails to distinguish between arrows with the same domain and range. More precisely, to any arrow $f \in \operatorname{Hom}(\mathcal{Q})$, there corresponds

\footnotetext{
${ }^{1} \mathrm{~A}$ 'small' category is one in which the collections of objects and arrows are genuine sets, rather than just classes.
} 
the arrow field $X_{f}$

$$
X_{f}(A):= \begin{cases}f & \text { if } \operatorname{Dom} f=A \\ \operatorname{id}_{A} & \text { otherwise }\end{cases}
$$

for all $A \in \mathrm{Ob}(\mathcal{Q})$. A representation is then said to 'separate arrows' (or 'distinguish arrows') if $\hat{a}\left(X_{f}\right) \neq \hat{a}\left(X_{g}\right)$ for all arrows $f, g \in \operatorname{Hom}(\mathcal{Q})$ with the same domain and range. This is manifestly false in the case of Eq. (1.1). Thus we have an incomplete representation of the categorial structure.

The main task in $\mathbf{I}$. was to develop a quantisation scheme in which arrows are separated. This involved choosing state vectors to be cross-sections of a Hilbert bundle $\mathcal{K}, A \rightsquigarrow \mathcal{K}[A]$, on $\mathrm{Ob}(\mathcal{Q})$, where the Hilbert spaces $\mathcal{K}[A]$ (which are strongly $A$-dependent) must be chosen so as to give an irreducible, faithful, representation of the category quantisation monoid. We will refer to this scheme as the 'Hilbert presheaf' approach since, as shown in $\mathbf{I} ., \mathcal{K}$ is actually a presheaf of Hilbert spaces.

For the special, and physically very important, case when $\mathcal{Q}$ is a category of finite sets (for example, causal sets), this scheme was discussed in detail in $[2]$ (hereafter referred to as II.) where it was argued that an appropriate choice for the Hilbert spaces $\mathcal{K}[A], A \in \mathrm{Ob}(\mathcal{Q})$, is ${ }^{2} \mathbb{C}^{|A|}$.

In the present paper we return to this example and rederive the results from a new perspective that further enhances their plausibility. This is a special case of a new approach in which the quantum states are complexvalued functions on the set of arrows in the category, rather than Hilbertbundle valued functions on the set of objects.

Having quantum states as functions on arrows has the advantage that it is easier to explore the problem of separating arrows with the same domain and range. In fact, the scheme can be viewed as an extension of the simple approach with complex-valued state functions $A \mapsto \psi(A)$ since a function $A \mapsto \psi(A)$ can be regarded as a special example of a function $h \mapsto \phi(h)$ in which $\phi$ depends on only the ranges ${ }^{3}$ of the arrows $h \in \operatorname{Hom}(\mathcal{Q})$.

The central idea is that by utilising aspects of an arrow in addition to its range, we can obtain a satisfactory quantum theory using only complexvalued state functions. In particular, arrows with the same domain and range - which are not separated by state vectors $A \mapsto \psi(A) \in \mathbb{C}$ - will be separated in the new approach. Of course, we anticipate that there should be some relation between this approach and the one based on a presheaf of

\footnotetext{
${ }^{2}|A|$ denotes the number of elements in the finite set $A$.

${ }^{3}$ Alternatively, $\phi$ can depend only on the domain of the arrows. However, choosing $\phi$ to depend on the ranges of the arrows allows for the neatest link with the ideas of $\mathbf{I}$.
} 
Hilbert spaces. In the important example of quantising on a category of sets we shall see explicitly that this is indeed the case.

The plan of the paper is as follows. In Section 2.1 we introduce the monoids of 'in' and 'out' arrow fields and show how they act on the set of objects in the category. This action is used in Section 2.2 to construct a representation of these monoids by operators acting on the space of complexvalued functions on $\operatorname{Hom}(\mathcal{Q})$. The formalism is extended in Section 2.3 to include configuration space operators.

The ensuing representation of the category quantisation monoid is highly reducible because of the action of the 'in' arrow fields and the action of a second copy of the space of configuration functions. This issue is addressed in Section 3 by considering how to reduce the number of arrows on which the state vectors are defined. We start in Section 3.1 by re-expressing the formalism in a way which emphasises that the state vectors can be written in the form $\psi(A, h)$ where $A \in \mathrm{Ob}(\mathcal{Q})$, and $h$ is any arrow whose range is $A$. This is used in Section 3.2 to motivate the introduction of a presheaf of arrows on $\mathcal{Q}$ which must be chosen such that the representation of the category quantisation monoid becomes irreducible, while remaining faithful (that is, while still separating arrows with the same domain and range).

As shown in Section 3.3, if the category $\mathcal{Q}$ has a terminal object 1 , a natural choice for this presheaf is to associate with each object $A$ the set of arrows $h: 1 \rightarrow A$; in other words, the set of global elements of $A$. By this means, we are able to reproduce the results obtained in II. (using a presheaf of Hilbert spaces) for the case when $\mathcal{Q}$ is a category of finite sets.

\section{$2 \quad$ Using The Monoid $\operatorname{AF}^{\text {in }}(\mathcal{Q}) \times \operatorname{AF}^{\text {out }}(\mathcal{Q})$}

\subsection{The Action of $\operatorname{AF}^{\text {in }}(\mathcal{Q}) \times \operatorname{AF}^{\text {out }}(\mathcal{Q})$ on $\operatorname{Hom}(\mathcal{Q})$}

In the first two papers in this series, I. and II., only 'out' arrow fields were used, where an 'out' arrow field $X$ is defined to be an association to each object $A \in \mathrm{Ob}(\mathcal{Q})$, of an arrow $X(A)$ whose domain is $A$. However, there is obviously another type of arrow field (an 'in' field) which associates to each object $A$ an arrow $Y(A)$ whose range is $A$. We shall denote by $\operatorname{AF}^{\text {in }}(\mathcal{Q})$ and $\operatorname{AF}^{\text {out }}(\mathcal{Q})$ the set of all 'in', and 'out', arrow fields respectively.

In I., the set $\operatorname{AF}^{\text {out }}(\mathcal{Q})$ was given a monoid structure ${ }^{4}$ by defining the product of $X_{1}, X_{2} \in \operatorname{AF}^{\text {out }}(\mathcal{Q})$ as

\footnotetext{
${ }^{4}$ The unit element is the arrow field $\iota$ defined by $\iota(A):=\mathrm{id}_{A}$ for all $A \in \operatorname{Ob}(\mathcal{Q})$.
} 


$$
X_{2} \& X_{1}(A):=X_{2}\left(\operatorname{Ran} X_{1}(A)\right) \circ X_{1}(A)
$$

for all $A \in \mathrm{Ob}(\mathcal{Q})$. In diagrammatic form, if $A \stackrel{X_{1}(A)}{\rightarrow} B \stackrel{X_{2}(B)}{\rightarrow} C$, then

$$
X_{2} \& X_{1}(A):=X_{2}(B) \circ X_{1}(A) .
$$

Similarly, if $Y_{1}, Y_{2} \in \operatorname{AF}^{\text {in }}(\mathcal{Q})$, we define

$$
Y_{2} \& Y_{1}(D):=Y_{1}(D) \circ Y_{2}\left(\operatorname{Dom} Y_{1}(D)\right)
$$

for all $D \in \mathrm{Ob}(\mathcal{Q})$. In diagrammatic form, if $F \stackrel{Y_{2}(E)}{\longrightarrow} E \stackrel{Y_{1}(D)}{\longrightarrow} D$, then

$$
Y_{2} \& Y_{1}(D):=Y_{1}(D) \circ Y_{2}(E) \text {. }
$$

The Hilbert presheaf quantisation scheme discussed in I. and II. is based on the action of the monoid $\operatorname{AF}^{\text {out }}(\mathcal{Q})$ on the set $\operatorname{Ob}(\mathcal{Q})$ given by

$$
\ell_{X}(A):=\operatorname{Ran} X(A)
$$

for all $X \in \operatorname{AF}^{\text {out }}(\mathcal{Q}), A \in \operatorname{Ob}(\mathcal{Q})$. Thus, if $X(A): A \rightarrow B$, then $\ell_{X}(A)=B$. This is an 'action' in the sense that (i) for each $X \in \operatorname{AF}^{\text {out }}(\mathcal{Q}), \ell_{X}$ is a transformation of $\mathrm{Ob}(\mathcal{Q})$ to itself; and (ii) for all $X_{1}, X_{2} \in \mathrm{Ob}(\mathcal{Q})$, we have $\ell_{X_{2}} \circ \ell_{X_{1}}=\ell_{X_{2} \& X_{1}}$.

The 'in' arrow fields also act on $\mathrm{Ob}(\mathcal{Q})$, with

$$
\varrho_{Y}(A):=\operatorname{Dom} Y(A)
$$

for all $Y \in \operatorname{AF}^{\text {in }}(\mathcal{Q}), A \in \mathrm{Ob}(\mathcal{Q})$. This might suggest that the product monoid $\operatorname{AF}^{\text {in }}(\mathcal{Q}) \times \operatorname{AF}^{\text {out }}(\mathcal{Q})$ acts on $\mathrm{Ob}(\mathcal{Q})$. However, this is not so since in general $\varrho_{Y}\left(\ell_{X}(A)\right) \neq \ell_{X}\left(\varrho_{Y}(A)\right)$, and hence the obvious transformations induced by pairs $(Y, X) \in \operatorname{AF}^{\text {in }}(\mathcal{Q}) \times \operatorname{AF}^{\text {out }}(\mathcal{Q})$ on $\mathrm{Ob}(\mathcal{Q})$ do not represent the monoid structure of $\operatorname{AF}^{\text {in }}(\mathcal{Q}) \times \operatorname{AF}^{\text {out }}(\mathcal{Q})$. This is why 'in' vector fields were not introduced into the quantum scheme described in I. and II.

However, the situation changes if we let the state functions be defined on arrows, rather than objects. For there is an obvious action of $\operatorname{AF}^{\mathrm{in}}(\mathcal{Q}) \times$ $\operatorname{AF}^{\text {out }}(\mathcal{Q})$ on $\operatorname{Hom}(\mathcal{Q})$, namely

$$
h \mapsto X(\operatorname{Ran} h) \circ h \circ Y(\operatorname{Dom} h)
$$

for all $h \in \operatorname{Hom}(\mathcal{Q})$ and $(Y, X) \in \operatorname{AF}^{\text {in }}(\mathcal{Q}) \times \operatorname{AF}^{\text {out }}(\mathcal{Q})$. In considering this expression it may be helpful to use the diagram

$$
E \stackrel{Y(D)}{\longrightarrow} D \stackrel{h}{\longrightarrow} R \stackrel{X(R)}{\longrightarrow} B .
$$




\subsection{Representing the 'In' and 'Out' Arrow Fields}

With the quantum state vectors now being taken as functions $\phi: \operatorname{Hom}(\mathcal{Q}) \rightarrow$ $\mathbb{C}$, we use the action in Eq. (2.7) to represent the arrow fields as follows. If $X \in \operatorname{AF}^{\text {out }}(\mathcal{Q})$, then we define (cf. Eq. (1.1))

$$
\begin{aligned}
(\hat{a}(X) \phi)(h) & :=\phi(X(\operatorname{Ran} h) \circ h) \\
& =\phi\left(l_{X(\operatorname{Ran} h)} h\right)
\end{aligned}
$$

and if $Y \in \operatorname{AF}^{\text {in }}(\mathcal{Q})$, then

$$
\begin{aligned}
(\hat{l}(Y) \phi)(h) & :=\phi(h \circ Y(\operatorname{Dom} h)) \\
& =\phi\left(r_{Y(\operatorname{Dom} h)} h\right)
\end{aligned}
$$

where the actions of $\operatorname{AF}^{\text {out }}(\mathcal{Q})$ and $\operatorname{AF}^{\text {in }}(\mathcal{Q})$ on $\operatorname{Hom}(\mathcal{Q})$ are defined from the transformation in Eq. (2.7) as

$$
\begin{aligned}
l_{X}(h) & :=X(\operatorname{Ran} h) \circ h \\
r_{Y}(h) & :=h \circ Y(\operatorname{Dom} h)
\end{aligned}
$$

for all $h \in \operatorname{Hom}(\mathcal{Q}), X \in \operatorname{AF}^{\text {out }}(\mathcal{Q})$ and $Y \in \operatorname{AF}^{\text {in }}(\mathcal{Q})$.

To see that these operators form genuine (anti-) representations of the appropriate monoids, suppose first that $X_{1}$ and $X_{2}$ are a pair of 'in' arrow fields which, for expository clarity, satisfy the diagram

$$
D \stackrel{h}{\longrightarrow} R \stackrel{X_{1}(R)}{\longrightarrow} B \stackrel{X_{2}(B)}{\longrightarrow} C .
$$

Then

$$
\begin{aligned}
\left(\hat{a}\left(X_{1}\right) \hat{a}\left(X_{2}\right) \phi\right)(h) & =\left(\hat{a}\left(X_{2}\right) \phi\right)\left(X_{1}(R) \circ h\right) \\
& =\phi\left(X_{2}(B) \circ\left(X_{1}(R) \circ h\right)\right)
\end{aligned}
$$

for all $h \in \operatorname{Hom}(\mathcal{Q})$. On the other hand,

$$
\left(\hat{a}\left(X_{2} \& X_{1}\right) \phi\right)(h)=\phi\left(\left(X_{2}(B) \circ X_{1}(R)\right) \circ h\right)
$$

and thus, using the associativity of arrow composition in the category $\mathcal{Q}$, we see that

$$
\hat{a}\left(X_{1}\right) \hat{a}\left(X_{2}\right)=\hat{a}\left(X_{2} \& X_{1}\right)
$$

as claimed.

Similarly, if $Y_{1}, Y_{2} \in \mathrm{AF}^{\mathrm{in}}(\mathcal{Q})$, then, with the aid of the diagram

$$
F \stackrel{Y_{2}(E)}{\longrightarrow} E \stackrel{Y_{1}(D)}{\longrightarrow} D \stackrel{h}{\longrightarrow} R
$$

we see that

$$
\begin{aligned}
\left(\hat{l}\left(Y_{1}\right) \hat{l}\left(Y_{2}\right) \phi\right)(h) & =\left(\hat{l}\left(Y_{2}\right) \phi\right)\left(h \circ Y_{1}(D)\right) \\
& =\phi\left(\left(h \circ Y_{1}(D)\right) \circ Y_{2}(E)\right) .
\end{aligned}
$$


On the other hand, Eq. (2.3) gives $\left(Y_{2} \& Y_{1}\right)(D)=Y_{1}(D) \circ Y_{2}(E)$, and so, for all $h \in \operatorname{Hom}(\mathcal{Q})$,

$$
\left(\hat{l}\left(Y_{2} \& Y_{1}\right) \phi\right)(h)=\phi\left(h \circ\left(Y_{1}(D) \circ Y_{2}(E)\right)\right) .
$$

Hence, for all $Y_{1}, Y_{2} \in \operatorname{AF}^{\text {in }}(\mathcal{Q})$,

$$
\hat{l}\left(Y_{1}\right) \hat{l}\left(Y_{2}\right)=\hat{l}\left(Y_{2} \& Y_{1}\right)
$$

as claimed.

Finally, with the aid of the diagram

$$
E \stackrel{Y(D)}{\longrightarrow} D \stackrel{h}{\longrightarrow} R \stackrel{X(R)}{\longrightarrow} B
$$

we see that, for all $X \in \operatorname{AF}^{\text {out }}(\mathcal{Q}), Y \in \operatorname{AF}^{\text {in }}(\mathcal{Q})$, and $h \in \operatorname{Hom}(\mathcal{Q})$,

$$
\begin{aligned}
(\hat{a}(X) \hat{l}(Y) \phi)(h) & =(\hat{l}(Y) \phi)(X(R) \circ h) \\
& =\phi((X(R) \circ h) \circ Y(D)) \\
& =\phi(X(R) \circ(h \circ Y(D))) \\
& =(\hat{a}(X) \phi)(h \circ Y(D)) \\
& =(\hat{l}(Y) \hat{a}(X) \phi)(h)
\end{aligned}
$$

and hence

$$
\hat{a}(X) \hat{l}(Y)=\hat{l}(Y) \hat{a}(X)
$$

for all $X \in \operatorname{AF}^{\text {out }}(\mathcal{Q})$ and $Y \in \operatorname{AF}^{\text {in }}(\mathcal{Q})$.

From Eq. (2.18), Eq. (2.22) and Eq. (2.25) it follows that we have an operator (anti-) representation of the monoid $\operatorname{AF}^{\text {in }}(\mathcal{Q}) \times \operatorname{AF}^{\text {out }}(\mathcal{Q})$.

\subsection{The Configuration Space Operators}

The next step is to represent the configuration-space functions $\beta: \mathrm{Ob}(\mathcal{Q}) \rightarrow$ $\mathbb{R}$. In the quantisation scheme based on sections of a bundle of Hilbert spaces $A \rightsquigarrow \mathcal{K}[A]$, a function $\beta \in F(\mathrm{Ob}(\mathcal{Q}), \mathbb{R})$ is represented by the operator $(\hat{\beta} \psi)(A):=\beta(A) \psi(A)$ or, in exponentiated form,

$$
(\hat{V}(\beta) \psi)(A):=e^{-i \beta(A)} \psi(A)
$$

and we need an analogue of this.

In the present context, there are two natural ways of representing a function $\beta$. Namely

$$
(\hat{\rho}(\beta) \phi)(h):=\beta(\operatorname{Ran} h) \phi(h)
$$


and

$$
(\hat{\lambda}(\beta) \phi)(h):=\beta(\operatorname{Dom} h) \phi(h) .
$$

Then, for all $h \in \operatorname{Hom}(\mathcal{Q}), X \in \operatorname{AF}^{\text {out }}(\mathcal{Q}$ ) (and denoting Ran $h$ by $R$ for typographical clarity), we have, using Eq. (2.9) and Eq. (2.27),

$$
\begin{aligned}
(\hat{a}(X) \hat{\rho}(\beta) \phi)(h) & =(\hat{\rho}(\beta) \phi)(X(R) \circ h) \\
& =\beta(\operatorname{Ran}\{X(R) \circ h\}) \phi(X(R) \circ h) \\
& =\beta(\operatorname{Ran} X(R)) \phi(X(R) \circ h)
\end{aligned}
$$

while, on the other hand,

$$
\begin{aligned}
(\hat{\rho}(\beta) \hat{a}(X) \phi)(h) & =\beta(\operatorname{Ran} h)(\hat{a}(X) \phi)(h) \\
& =\beta(R) \phi(X(R) \circ h) .
\end{aligned}
$$

Now, if the function $\beta$ is replaced with the function $\beta \circ \ell_{X}: \operatorname{Ob}(\mathcal{Q}) \rightarrow \mathbb{R}$, the right side of Eq. (2.30) now contains $\beta\left(\ell_{X} R\right)$. But, by the definition of $\ell_{X}$, we have $\ell_{X} R=\operatorname{Ran} X(R)$, and hence

$$
\hat{a}(X) \hat{\rho}(\beta)=\hat{\rho}\left(\beta \circ \ell_{X}\right) \hat{a}(X) .
$$

Thus, if $\hat{V}(\beta):=\exp -i \hat{\rho}(\beta)$, we have a representation of the category quantisation monoid $\operatorname{AF}^{\text {out }}(\mathcal{Q}) \times_{\ell} F(\mathrm{Ob}(\mathcal{Q}), \mathbb{R})$.

Next, we must consider the implications of the second beta operator, $\hat{\lambda}(\beta)$, defined in Eq. (2.28). If $Y \in \operatorname{AF}^{\text {in }}(\mathcal{Q})$ then, for all $h \in \operatorname{Hom}(\mathcal{Q})$ (and denoting Dom $h$ by $D$ for typographical clarity), we have

$$
\begin{aligned}
(\hat{\lambda}(\beta) \hat{l}(Y) \phi)(h) & =\beta(D)(\hat{l}(Y) \phi)(h) \\
& =\beta(D) \phi(h \circ Y(D))
\end{aligned}
$$

while

$$
\begin{aligned}
(\hat{l}(Y) \hat{\lambda}(\beta) \phi)(h) & =(\hat{\lambda}(\beta) \phi)(h \circ Y(D)) \\
& =\beta(\operatorname{Dom}[h \circ Y(D)]) \phi(h \circ Y(D)) \\
& =\beta(\operatorname{Dom} Y(D)) \phi(h \circ Y(D)) .
\end{aligned}
$$

However, the action of the 'in' arrow field $Y$ on $\mathrm{Ob}(\mathcal{Q})$ is $\varrho_{Y}: A \mapsto$ $\operatorname{Dom} Y(A)$, and hence, from Eq. (2.32), it follows that, for all $h \in \operatorname{Hom}(\mathcal{Q})$

$$
\begin{aligned}
\left(\hat{\lambda}\left(\beta \circ \varrho_{Y}\right) \hat{l}(Y) \phi\right)(h) & =\left(\beta \circ \varrho_{Y}\right)(D) \phi(h \circ Y(D)) \\
& =\beta(\operatorname{Dom} Y(D)) \phi(h \circ Y(D)) .
\end{aligned}
$$

Thus

$$
\hat{\lambda}\left(\beta \circ \varrho_{Y}\right) \hat{l}(Y)=\hat{l}(Y) \hat{\lambda}(\beta)
$$

which, defining $\hat{W}(\beta):=\exp -i \hat{\lambda}(\beta)$, corresponds to an operator representation of the monoid $\operatorname{AF}^{\text {in }}(\mathcal{Q}) \times \varrho F(\operatorname{Ob}(\mathcal{Q}), \mathbb{R})$. 
Furthermore, if $X \in \operatorname{AF}^{\text {out }}(\mathcal{Q})$ and $\beta \in F(\mathrm{Ob}(\mathcal{Q}), \mathbb{R})$, then (again with $h: D \rightarrow R)$

$$
\begin{aligned}
(\hat{a}(X) \hat{\lambda}(\beta) \phi)(h) & =(\hat{\lambda}(\beta) \phi)(X(R) \circ h) \\
& =\beta(\operatorname{Dom} X(R) \circ h) \phi(X(R) \circ h) \\
& =\beta(D) \phi(X(R) \circ h) .
\end{aligned}
$$

However,

$$
\begin{aligned}
(\hat{\lambda}(\beta) \hat{a}(X) \phi)(h) & =\beta(D)(\hat{a}(X) \phi)(h) \\
& =\beta(D) \phi(X(R) \circ h)
\end{aligned}
$$

and so

$$
[\hat{a}(X), \hat{\lambda}(\beta)]=0
$$

Of course, this could have been expected since the action of $\hat{a}(X)$ involves attaching arrows to the range of $h$, whereas $\hat{\lambda}(\beta)$ acts only on the domain of $h$.

Similarly, it is easy to show that

$$
[\hat{\rho}(Y), \hat{l}(\beta)]=0 .
$$

In addition, it is obvious that, for all $\beta_{1}, \beta_{2} \in F(\mathrm{Ob}(\mathcal{Q}), \mathbb{R})$,

$$
\left[\hat{\rho}\left(\beta_{1}\right), \hat{\lambda}\left(\beta_{2}\right)\right]=0
$$

which, together with Eq. (2.25), Eq. (2.38) and Eq. (2.39), implies that we have an operator representation of the monoid $\left(\operatorname{AF}^{\text {out }}(\mathcal{Q}) \times_{\ell} F(\mathrm{Ob}(\mathcal{Q}), \mathbb{R})\right) \times$ $\left(\operatorname{AF}^{\mathrm{in}}(\mathcal{Q}) \times{ }_{\varrho} F(\mathrm{Ob}(\mathcal{Q}), \mathbb{R})\right)$.

\section{$3 \quad$ Reducing the Number of Arrows}

\subsection{Rewriting the Formalism}

The representation constructed above of the category quantisation monoid $\operatorname{AF}^{\text {out }}(\mathcal{Q}) \times_{\ell} F(\mathrm{Ob}(\mathcal{Q}), \mathbb{R})$ certainly separates the arrows of $\mathcal{Q}$. Indeed, if $f, g: A \rightarrow B$, then (cf. Eq. (1.7))

$$
\left(\hat{a}\left(X_{f}\right) \phi\right)\left(\operatorname{id}_{A}\right)=\phi(f)
$$

and

$$
\left(\hat{a}\left(X_{g}\right) \phi\right)\left(\mathrm{id}_{A}\right)=\phi(g)
$$

which, since there are certainly functions $\phi$ such that $\phi(f) \neq \phi(g)$, shows that $\hat{a}\left(X_{f}\right) \neq \hat{a}\left(X_{g}\right)$. 
However, there are two problems with this representation. The first is that in order to construct a proper Hilbert space for the quantum states $\phi: \operatorname{Hom}(\mathcal{Q}) \rightarrow \mathbb{C}$, we need a measure $\mu$ on $\operatorname{Hom}(\mathcal{Q})$, so that the inner product between two states $\phi$ and $\psi$ can be defined as

$$
\langle\phi, \psi\rangle:=\int_{\operatorname{Hom}(\mathcal{Q})} d \mu(h) \phi(h)^{*} \psi(h) .
$$

This is as challenging as the corresponding problem in the Hilbert presheaf scheme of finding measures on $\operatorname{Ob}(\mathcal{Q})$. If $\operatorname{Hom}(\mathcal{Q})$ is finite, or countably infinite, the obvious discrete measures can be used. In other situations, a more detailed investigation is needed, and I hope to return to this in a later paper.

The second problem is that because the quantum states carry a representation of the monoid $\left(\operatorname{AF}^{\text {out }}(\mathcal{Q}) \times{ }_{\ell} F(\mathrm{Ob}(\mathcal{Q}), \mathbb{R})\right) \times\left(\mathrm{AF}^{\text {in }}(\mathcal{Q}) \times_{\varrho} F(\mathrm{Ob}(\mathcal{Q}), \mathbb{R})\right)$ even if this representation is irreducible, the associated representation of the category quantisation monoid $\operatorname{AF}^{\text {out }}(\mathcal{Q}) \times_{\ell} F(\mathrm{Ob}(\mathcal{Q}), \mathbb{R})$ will generally not be.

One obvious solution is to reduce the number of arrows on which the state functions are defined, and to help in this task it is useful to think of the states we have introduced as functions $\phi(R, h)$ where $R \in \mathrm{Ob}(\mathcal{Q})$ and $h \in \operatorname{Hom}(-, R)$, the set of all arrows whose range is $R$. In this notation, the operators defined above are as follows, where $X \in \operatorname{AF}^{\text {out }}(\mathcal{Q}), Y \in \operatorname{AF}^{\text {in }}(\mathcal{Q})$, $\beta_{1}, \beta_{2} \in F(\mathrm{Ob}(\mathcal{Q}), \mathbb{R})$ :

$$
\begin{aligned}
(\hat{a}(X) \phi)(R, h) & :=\phi(B, X(R) \circ h) \\
\left(\hat{\rho}\left(\beta_{1}\right) \phi\right)(h) & :=\beta_{1}(R) \phi(R, h) \\
(\hat{l}(Y) \phi)(R, h) & :=\phi(R, h \circ Y(D)) \\
\left(\hat{\lambda}\left(\beta_{2}\right) \phi\right)(R, h) & :=\beta_{2}(D) \phi(R, h)
\end{aligned}
$$

for all $R \in \mathrm{Ob}(\mathcal{Q})$ and $h \in \operatorname{Hom}(-, R)$. Once again, for typographical clarity we are using the diagram

$$
E \stackrel{Y(D)}{\longrightarrow} D \stackrel{h}{\longrightarrow} R \stackrel{X(R)}{\longrightarrow} B .
$$

Of course, the ' $R$ ' label is not really necessary, since the arrow $h$ already 'knows' what its range is. However, by writing the operator definitions in this form it is clear that the action of the 'out' arrow fields is on the objects in $\mathcal{Q}$ - as in the Hilbert presheaf scheme - but now supplemented by an action on the arrows that come into each object. It is also clear how the extra operators (i.e., those outside the category quantisation monoid $\left.\operatorname{AF}^{\text {out }}(\mathcal{Q}) \times_{\ell} F(\mathrm{Ob}(\mathcal{Q}), \mathbb{R})\right) \hat{l}(Y)$ and $\hat{\lambda}(\beta)$ act on the 'left hand end' of the arrow $h \in \operatorname{Hom}(-, R)$. It is the existence of these operators that is 
responsible for the reducible nature of the representation of $\operatorname{AF}^{\text {out }}(\mathcal{Q}) \times_{\ell}$ $F(\mathrm{Ob}(\mathcal{Q}), \mathbb{R})$.

\subsection{Introducing Presheaves of Arrows}

As remarked above, to get an irreducible representation of $\operatorname{AF}^{\text {out }}(\mathcal{Q}) \times_{\ell}$ $F(\mathrm{Ob}(\mathcal{Q}), \mathbb{R})$ the number of arrows that enter each object must be restricted, albeit in such a way that the quantisation scheme still separates arrows. Thus we are interested in subsets of arrows $J[R] \subset \operatorname{Hom}(-, R)$ so that we can define $\hat{a}(X)$ as (cf. Eq. (3.4))

$$
(\hat{a}(X) \phi)(R, h):=\phi(\operatorname{Ran} X(R), X(R) \circ h)
$$

for all $h \in J[R], R \in \mathrm{Ob}(\mathcal{Q})$. Clearly, for Eq. (3.9) to be consistent we require that if $h \in J[R]$ and $f: R \rightarrow B$, then $f \circ h \in J[B]$.

To understand better what this condition means, note that the association $A \rightsquigarrow \operatorname{Hom}(-, A)$ defines a covariant presheaf $\mathbf{H}$ on $\mathcal{Q}$; i.e., $\mathbf{H}$ is a covariant functor from the category $\mathcal{Q}$ to the category of sets Set. More precisely:

i) to each object $A$ in $\mathcal{Q}$, the functor $\mathbf{H}$ associates the set $\operatorname{Hom}(-, A)$ of all arrows whose range is $A$;

ii) to each arrow $f: A \rightarrow B$, the functor $\mathbf{H}$ associates the function $H(f): \operatorname{Hom}(-, A) \rightarrow \operatorname{Hom}(-, B)$ defined by

$$
H(f)(h):=f \circ h
$$

for all $h \in \operatorname{Hom}(-, A)$.

Then the condition above on the sets $J[A] \subset \operatorname{Hom}(-, A), A \in \operatorname{Ob}(\mathcal{Q})$, means that the association $A \rightsquigarrow J[A]$ corresponds to a presheaf $\mathbf{J}$ that is a subobject of $\mathbf{H}$ in the category $\mathcal{Q}^{\text {Set }}$ of (covariant) presheaves on $\mathcal{Q}$.

In the (topos) category $\mathcal{Q}^{\text {Set }}$, the sub-objects of a presheaf $\mathbf{H}$ are in oneto-one correspondence with arrows in the category $\mathcal{Q}^{\text {Set }}$

$$
\chi_{J}: \mathbf{H} \rightarrow \mathbf{\Omega}
$$

where $\boldsymbol{\Omega}$ is the presheaf of sieves on $\mathcal{Q}$. Here, we recall that a sieve on an object $A \in \mathrm{Ob}(\mathcal{Q})$ is defined to be a set $S$ of arrows in $\mathcal{Q}$ whose domain is $A$ (thus $S \subset \operatorname{Hom}(A,-)$ ) and with the property that if $f \in S$ then $k \circ f \in S$ for all arrows $k$ such that Dom $k=\operatorname{Ran} f$ (thus, in a poset category, a sieve is just an upper set) [3]. We denote by $\Omega[A]$ the set of all sieves on $A$. If $f: A \rightarrow B$, the map $\Omega(f): \Omega[A] \rightarrow \Omega[B]$ is defined by

$$
\Omega(f)(S):=\{g \in \operatorname{Hom}(B,-) \mid g \circ f \in S\}
$$


for all $S \in \Omega[A]$.

The arrow $\chi_{J}$ in Eq. (3.11) associates to each $A \in \mathrm{Ob}(\mathcal{Q})$, a map $\chi_{J}[A]$ : $\operatorname{Hom}(-, A) \rightarrow \Omega[A]$ which is defined by saying that if $h \in \operatorname{Hom}(-, A)$ then $\chi_{J}[A](h)$ is the set of all arrows $k$ with domain $A$ such that $k \circ h$ belongs to $J[\operatorname{Ran} k]$. It is easy to see that these form a sieve on $A$.

The challenge is to find a presheaf $\mathbf{J}$ of arrows such that the associated representation of the category quantisation monoid is irreducible whilst maintaining the separation of arrows in the quantum scheme. Such a presheaf can be used in two ways:

1) $\mathbf{J}$ can be used as it stands. The states are then functions $\phi(R, h)$, where $h \in J[R]$ is an arrow whose range is $R \in \mathrm{Ob}(\mathcal{Q})$.

2) However, if desired certain arrows can be added to the domain of the state vectors in addition to those in the subsets $J[A], A \in \mathrm{Ob}(\mathcal{Q})$. To see this, for each object $R$ consider the arrows $h \in \operatorname{Hom}(-, R)$ that are 'picked out' by $\chi_{J}[R]$ in the sense that there exists $B \in \mathrm{Ob}(\mathcal{Q})$ and an arrow $k: R \rightarrow B$, such that $k \circ h \in J[B]$.

Now, using Dirac notation, when $\hat{a}(X)^{\dagger}$ acts on a state $|B, m\rangle$, $m \in \operatorname{Hom}(-, B)$ it takes it into a linear combination of states $|R, h\rangle$, $h \in \operatorname{Hom}(-, R)$, with the property that (i) $X(R): R \rightarrow B$; and (ii) $X(R) \circ h=m .{ }^{5} \quad$ If the extra arrows 'picked out' by $\chi_{J}$ are added to the domain of the state vectors, then if $m \in J[B]$ this sum would include all the states $|R, h\rangle(R \in \operatorname{Ob}(\mathcal{Q}) h \in \operatorname{Hom}(-, R))$ such that $\chi_{J}[R](h)=m$.

\subsection{The Presheaf of Global Elements of the Objects of $\mathcal{Q}$}

We will now discuss one particularly important example of a presheaf $\mathbf{J}$. This is motivated by remarking, once again, that the representation of the category quantisation monoid $\operatorname{AF}^{\text {out }}(\mathcal{Q}) \times{ }_{\ell} F(\mathrm{Ob}(\mathcal{Q}), \mathbb{R})$ is reducible because of the existence of the operators defined in Eq. (3.6) and Eq. (3.7) as

$$
\begin{aligned}
(\hat{l}(Y) \phi)(R, h) & :=\phi(R, h \circ Y(D)) \\
(\hat{\lambda}(\beta) \phi(R, h) & :=\beta(D) \phi(R, h)
\end{aligned}
$$

where once again we have used the diagram

$$
E \stackrel{Y(D)}{\longrightarrow} D \stackrel{h}{\longrightarrow} R \stackrel{X(R)}{\longrightarrow} B
$$

for typographical clarity.

\footnotetext{
${ }^{5}$ This is the analogue of the results on the adjoints of operators obtained in $\mathbf{I}$.
} 
One way of trivialising these operators that latch onto the 'left hand end' of the arrows $h$ is available if there is a terminal object ${ }^{6} 1$ in the category $\mathcal{Q}$. In this case, for all $R \in \mathrm{Ob}(\mathcal{Q})$, we can try restricting the domains of the arrows in $J[R]$ to be 1 , so that each arrow $h \in J[R]$ is of the form $h: 1 \rightarrow R$. In other words, $h$ is a global element of the object $R$. Thus $J[R]$ is defined to be the set of all global elements of $R$. Note that, if $h: 1 \rightarrow R$ is a global element of $R$, then $X(R) \circ h: 1 \rightarrow B$, and hence $X(R) \circ h$ is a global element of $B$. Thus the association $A \rightsquigarrow J[A]$ defines a genuine presheaf $\mathbf{J}$ that is a subobject of the presheaf $\mathbf{H}$.

This choice for $\mathbf{J}$ works because, since there is only one arrow from 1 to itself (i.e., $\left.\mathrm{id}_{1}\right)$ the only 'in' arrow field that can act on an arrow $h: 1 \rightarrow R$, is the identity. Similarly, the operator $\hat{\lambda}(\beta)$ is now

$$
(\hat{\lambda}(\beta) \phi)(R, h)=\beta(1) \phi(R, h)
$$

for all $R \in \mathrm{Ob}(\mathcal{Q})$ and $h \in J[R]$. This can safely be ignored since it just multiplies each state vector by the same complex number.

Thus we are left with the operators

$$
\begin{aligned}
(\hat{a}(X) \phi)(R, h) & :=\phi(B, X(R) \circ h) \\
(\hat{V}(\beta) \phi)(R, h) & :=e^{-i \beta(R)} \phi(R, h)
\end{aligned}
$$

for all $R \in \mathrm{Ob}(\mathcal{Q})$, and all $h: 1 \rightarrow R$. This is certainly a representation of the category quantisation monoid, and so the important question now is whether it separates arrows. Clearly this will be so provided, if $f, g: R \rightarrow B$, there exists $h: 1 \rightarrow R$ such that $f \circ h \neq g \circ h$.

The existence of such separating arrows depends on the category. For example, if $\mathcal{Q}$ is a category of sets then everything works. This is because the global elements of a set are just its elements in the normal sense-so that $J[A] \simeq A$ for all $A \in \mathrm{Ob}(\mathcal{Q})$-and, of course, a function between two sets is completely specified by its action on these elements. Thus, in this situation, the quantum states are functions $\phi(A, a)$, where $a \in A$, and basic operators are

$$
\begin{aligned}
(\hat{a}(X) \phi)(A, a) & :=\phi(B, X(A)(a)) \\
(\hat{V}(\beta) \phi)(A, a) & :=e^{-i \beta(A)} \phi(A, a)
\end{aligned}
$$

for all $a \in A$, and where $X(A)$ is now a function from the set $A$ to the set $B$.

\footnotetext{
${ }^{6}$ A terminal object in a category $\mathcal{Q}$ is an object 1 with the property that, for each object $A$ in $\mathcal{Q}$, there is precisely one arrow $1_{A}: A \rightarrow 1$. It is easy to see that any two terminal objects are isomorphic.
} 
However, Eqs. (3.19-3.20) essentially reproduce the Hilbert presheaf representation discussed in detail in $\mathbf{I I}$. There, the Hilbert space $\mathcal{K}[A]$ was identified with $\mathbb{C}^{|A|}, A \in \mathrm{Ob}(\mathcal{Q})$, and this is isomorphic to the space of complexvalued functions on $A$. Furthermore, the representation of $X \in \operatorname{AF}^{\text {out }}(\mathcal{Q})$ by the operator $\hat{a}(X)$ in Eq. (3.19) reproduces the representation by the corresponding operator in the presheaf of Hilbert spaces. Thus the approach developed above further justifies the choice made in II. of the Hilbert presheaf for quantising on a category of sets.

In fact, these considerations suggests a general way of relating the structure in the present paper with that developed in I. and II. Specifically, if $\mathbf{J}$ is a presheaf of arrows, the state vectors are functions $\phi(R, h)$ where $R \in \mathrm{Ob}(\mathcal{Q})$ and $h \in J[R] \subset \operatorname{Hom}(-, R)$. Thus each fixed $R$ gives a function $\phi_{R}: J[R] \rightarrow \mathbb{C}$ defined as $\phi_{R}(h):=\phi(R, h)$ for all $h \in J[R]$. Now, for each $A \in \mathrm{Ob}(\mathcal{Q})$, let $\mathcal{K}[A]$ be the set, $F(J[A], \mathbb{C})$, of all complex-valued functions on $J[A]$; and if $f: A \rightarrow B$ define $\kappa(f): \mathcal{K}[B] \rightarrow \mathcal{K}[A]$ by

$$
(\kappa(f) v)(h):=v(f \circ h)
$$

for all $v \in F(J[B], \mathbb{C})$, and $h \in J[A]$. It is easy to check that these assignments of $\mathcal{K}[A], A \in \mathrm{Ob}(\mathcal{Q})$, and $\kappa(f): \mathcal{K}[B] \rightarrow \mathcal{K}[A]$ constitute a presheaf of vector spaces over $\mathcal{Q}$. Thus we regain the structure discussed in I. and II. Of course, we need to worry about putting inner products on these vector spaces, but that is closely related to the question of measures on $\operatorname{Hom}(\mathcal{Q})$, which is a subject for future research.

\section{Conclusion}

We have introduced an approach to quantising on a category that provides an alternative to the techniques used in the earlier papers I. and II. The new scheme involves defining quantum states to be complex-valued functions on the set of arrows in the category $\mathcal{Q}$, rather than cross-sections of a bundle/presheaf of Hilbert spaces over the set of objects in $\mathcal{Q}$.

Arguably, in some respects this new approach is more transparent than the original one; in any event, it certainly provides a new way of looking at things. For example, it throws light on the choice of Hilbert-space presheaves used in the original approach; particularly when $\mathcal{Q}$ is a category of sets. In general, in the new approach we need to find presheaves of arrows, which in some respects is a simpler thing to do. For a category of structured sets (for example: topological spaces; causal sets), this may help with the problem of finding presheaves that distinguish only the structure-preserving maps between sets. 
From a mathematical perspective, one of the main problems is to find suitable measure structures on the space of arrows $\operatorname{Hom}(\mathcal{Q})$ in $\mathcal{Q}$; or on the appropriate subspace of $\operatorname{Hom}(\mathcal{Q})$ if a sub-sheaf of arrows is selected. This is not dissimilar to the problem of constructing measures on the set of objects, $\mathrm{Ob}(\mathcal{Q})$, and is something to which I hope to return later.

\section{Acknowledgements}

Support by the EPSRC in form of grant GR/R36572 is gratefully acknowledged. I am very grateful to Jeremy Butterfield for a critical reading of the manuscript.

\section{References}

[1] C. J. Isham, A new approach to quantising space-time: I. Quantising on a general category. gr-qc/0303060 (2003).

[2] C. J. Isham, A new approach to quantising space-time: II. Quantising on a category of sets. gr-qc/0304077 (2003).

[3] S. MacLane and I. Moerdijk, Sheaves in Geometry and Logic: A First Introduction to Topos Theory. Springer-Verlag, London, (1992). 